\title{
BIOSYNTHESIS OF COPPER NANOPARTICLES USING PARTHENIUM HYSTEROPHORUS LEAF EXTRACT AND SCREENING ITS ANTIMICROBIAL ACTIVITY
}

\author{
ROHINI SAHAYA MARY ${ }^{1 *}$, MAHESH MK ${ }^{2}$, PRUTHVI ML ${ }^{2}$ \\ ${ }^{1}$ Department of Botany, Teresian College, Mysore, Karnataka, India. ${ }^{2}$ Department of Botany, Yuvaraja's College, Mysore, Karnataka, India. \\ Email: srrohi@gmail.com
}

Received: 14 October 2019, Revised and Accepted: 15 November 2019

\section{ABSTRACT}

Objective: The aim of this study is to synthesis the copper nanoparticles (CuNPs) using the leaf extract of Parthenium hysterophorus.

Methods: Dry and fresh leaf extract was prepared and $\mathrm{CuSo}_{4}$ was added. The color change was noted and recorded by ultraviolet-visible spectrophotometer. The morphological characteristics were analyzed by scanning electron microscopy (SEM). Antimicrobial activities were performed by the disc diffusion method.

Results: The color change indicates the production of CuNPs. Surface plasmon resonance band was observed around $599 \mathrm{~nm}$ and $572 \mathrm{~nm}$ for fresh and dry samples of P. hysterophorus leaf extract. SEM confirms the formation and the crystalline nature of CuNPs and X-ray diffraction studies show the particle size. The antibacterial potentials of the CuNPs were studied and have shown good high inhibition activity against Staphylococcus aureus, Bacillus subtilis, Proteus vulgaris, and Pseudomonas aeruginosa at different concentrations in compare to fungi species.

Conclusion: This method is effective and environmental friendly for the synthesis of CuNPs using leaf extract of Parthenium hysterophorus.

Keywords: Copper nanoparticles, Parthenium hysterophorus, Antimicrobial activity, Scanning electron microscopy, X-ray diffraction

(C) 2020 The Authors. Published by Innovare Academic Sciences Pvt Ltd. This is an open access article under the CC BY license (http://creativecommons. org/licenses/by/4. 0/) DOI: http://dx.doi.org/10.22159/ajpcr.2020.v13i1.36076

\section{INTRODUCTION}

Nanotechnology is mainly concerned with the synthesis of nanoparticles of variable sizes, shapes, chemical compositions, and controlled disparity and their potential use for human benefits. Although chemical and physical methods may successfully produce pure, well-defined nanoparticles, these are quite expensive and potentially dangerous to the environment. The use of biological organisms such as microorganisms, plant extracts, or plant biomass could be an alternative to chemical and physical methods for the production of nanoparticles in an eco-friendly manner [1]. Plant extracts may act both as reducing agents and stabilizing agents in the synthesis of nanoparticles. The source of the plant extract is known to influence the characteristics of the nanoparticles [2]. This is because different extracts contain different concentrations and combinations of organic reducing agents [3]. Typically, a plant extract-mediated bioreduction involves mixing the aqueous extract with an aqueous solution of the relevant metal salt. Nanoparticle bound drugs have an extended half-life in vivo, longer circulation times and can convey a high concentration of a potent drug to where it is needed [4]. The size of the drug nanoparticle and its surface characteristics can be modified to achieve the desired delivery characteristics [5]. As the nanoparticle-bound drug is not able to circulate broadly, its side effects are reduced and a high localized concentration can be achieved where it is needed [6]. In view of the large surface area per unit mass of nanoparticles, the drug loading can be relatively high [7]. Nanoparticle-bound drugs are easily suspended in liquids and are able to penetrate deep in organs and tissues. Human beings have been using copper $(\mathrm{Cu})$ and $\mathrm{Cu}$ complexes for various purposes for centuries, such as water purifiers, algaecides, fungicides, and antibacterial and antifouling agents [8]. Natural plant materials such as aqueous extracts of fresh and dry leaf of Parthenium hysterophorus have been used for the synthesis of $\mathrm{Cu}$ nanoparticles. The application of nanoparticles expresses superior antibacterial activity against bacteria and fungi. The nanoparticles have been used as non-toxic aqueous formulations for the administration of cancer therapy [9]. $\mathrm{Cu}$ and $\mathrm{CuO}$ nanoparticles have been studied as potential antimicrobial agents against infectious organisms such as Escherichia coli, Bacillus subtilis, Vibrio cholera, Pseudomonas aeruginosa, syphilis typhus, and Staphylococcus aureus [10]. Biosynthesis of metal nanoparticles by plants is currently under development. The synthesis of metal nanoparticles using inactivated plant tissue, plant extracts, exudates, and other parts of living plants is a modern alternative for their production [11]. It is a very cost-effective method and, therefore, a prospective commercial alternative for large-scale production. The present work details a green chemistry approach to the synthesis of metal oxide nanoparticles using plant extract which acts as a reducing agent. In this current research paper, $\mathrm{CuO}$ nanoparticles have been synthesized and characterized by scanning electron microscope (SEM) and X-ray diffraction (XRD) analysis. Further, $\mathrm{CuO}$ nanoparticles were explored with respect to their prospective antimicrobial applications.

\section{METHODS}

Collection of plant material

P. hysterophorus was collected from Mysore, Karnataka. The plant specimen was identified and authenticated by Dr. Ganesh Babu from the Foundation for Revitalization of Local Health Traditions, Bengaluru (NMGB 106918).

\section{Preparation of fresh and dry plant extracts}

The leaves of $P$. hysterophorus were washed under tap water to remove dust and other particles. The well-cleansed leaves were cut into small pieces and weighed up to $20 \mathrm{~g}$. Leaves were boiled in $100 \mathrm{ml}$ of distilled water for $15 \mathrm{~min}$. The plant extract was filtered using the Whatman filter paper. The dry plant extract was prepared by drying the leaves under the shade at room temperature. The finely powdered leaves were boiled and filtered. 
Synthesis of copper nanoparticles (CuNPs)

About $5 \mathrm{ml}$ of fresh and dry extract was added to $25 \mathrm{ml}$ of $1 \%$ aqueous copper sulfate in two different conical flasks and stirred for constant mixing. It was incubated for $24 \mathrm{~h}$ at room temperature. A color change of the solutions was noted by visual inspection confirming the synthesis of CuNPs.

\section{Ultraviolet (UV)-Visible spectral analysis}

The bioreduction of the $\mathrm{Cu}$ (II) in the aqueous solution was monitored by measuring the solution on a UV-visible spectrophotometer in 200-800 nm using $1 \mathrm{ml}$ of sample and compared with $1 \mathrm{ml}$ of distilled water which is used as a blank.

\section{XRD}

The particle size and nature of the CuNPs were determined using Bruker Eco D8 Advance X'pert PRO operating at a voltage of $40 \mathrm{kV}$, a current of $20 \mathrm{~mA}$ with copper $\mathrm{K} \alpha$ radiation at $2 \theta$ angle ranging from $10^{*}$ to $80^{*}$. A thin film of the CuNP was made by dipping a glass plate in a solution and carried out for XRD studies. The crystalline CuNP was calculated from the width of the XRD peaks and the average size of the nanoparticles can be estimated using the Debye Scherrer equation, $\mathrm{D}=\mathrm{k} \lambda / \beta \cos \theta$.

\section{SEM}

The morphological feature of synthesized CuNP from dry and fresh leaf extracts of $P$. hysterophorus was studied by SEM. The suspension of nanoparticle was dried into powder and about $1 \mathrm{mg}$ fine powder was used for the SEM analysis. SEM analysis was carried out on fine coater for uniform coating of platinum on the sample. Then, the samples were characterized in the SEM at an accelerating voltage of $15 \mathrm{kV}$.

\section{Antibacterial activity}

The synthesized CuNPs were examined against pathogenic bacteria such as $S$. aureus, B. subtilis, Proteus vulgaris, and P. aeruginosa by the agar disc diffusion method. The pure bacterial cultures were subcultured on nutrient broth. The broth was swabbed on a culture plate homogeneously using a sterile L-shaped glass rod. The sterile discs dipped in different concentrations of CuNPs $(0.2,0.4,0.6,0.8$, and 1.0) were placed on the nutrient agar plate. Tetracycline, the standard drug was used as a control. The treated plates were then incubated at $37^{\circ} \mathrm{C}$ for $24 \mathrm{~h}$. The antibacterial action of CuNPs was evaluated by the extent of the zone of inhibition.

\section{Antifungal activity}

The selected fungi such as Penicillium spp., Fusarium oxysporum, and Aspergillus niger were tested against the synthesized CuNPs by the agar disc diffusion method. The fungal cultures were subcultured on nutrient broth. The broth was swabbed on a culture plate homogeneously using a sterile L-shaped glass rod. The sterile discs dipped in different concentrations of CuNPs $(10,20,30,40$, and 50$)$ were placed on the potato dextrose agar plate. Fluconazole, the standard drug was used as a control. The treated plates were then incubated at $37^{\circ} \mathrm{C}$ for 24-48 $\mathrm{h}$ [12]. The antifungal action of CuNPs was evaluated by the extent of the zone of inhibition.

\section{RESULTS}

\section{Synthesis of CuNPs}

The indication of the synthesis of CuNPs was observed by the color change in the plant extract. It was observed with time, the color of the reaction mixture changed from light yellow, as shown in Fig. 1.

\section{UV-visible spectrophotometer analysis}

UV-visible spectrophotometer was used to identify the synthesis of CuNPs. The reduction of copper ions into CuNPs in the presence of Parthenium leaf extracts was observed as a result of the color change. The color change is due to the surface plasmon resonance (SPR) phenomenon. SPR is the resonant oscillation of conduction electrons at the interface between negative and positive permittivity material stimulated by incident light. The metal nanoparticles such as CuNPs have free electrons which give the SPR absorption band due to the combined vibration of electrons of metal nanoparticles in resonance with light wave. The sharp bands of CuNPs were observed around $599 \mathrm{~nm}$ and $572 \mathrm{~nm}$ for fresh and dry samples of $P$. hysterophorus leaf extract (Fig. 2). This confirms that the plant leaf extracts (both dry and fresh) have the potential to reduce copper ions to CuNPs.

\section{XRD}

XRD obtained for CuNPs of dry and fresh $P$. hysterophorus leaf extracts showed a characteristic peak near 2-theta values of dry leaf extract at $64.74 \mathrm{~nm}$ (Fig. 3) and fresh leaf extract at $64.05 \mathrm{~nm}$ (Fig. 4). The XRD pattern thus shows that CuNPs were crystalline in nature. In addition to the Bragg peak representative of copper nanocrystals, additional and yet unassigned peaks were also observed, suggesting that the

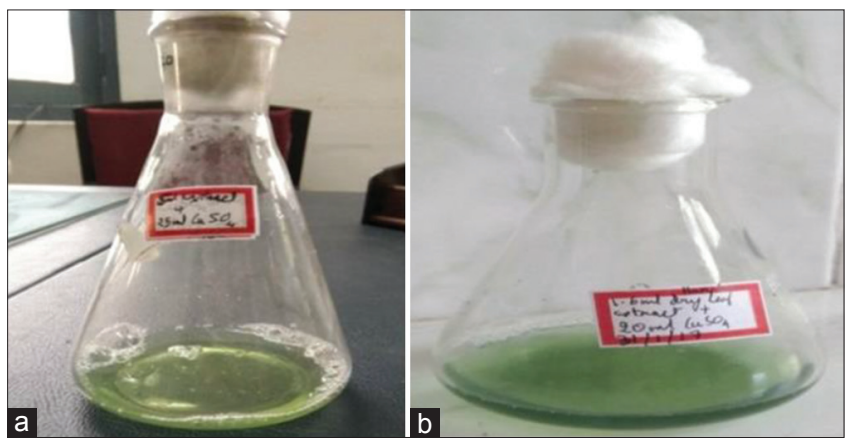

Fig. 1: Parthenium fresh (a) and dry (b) leaf extracts copper nanoparticle solution
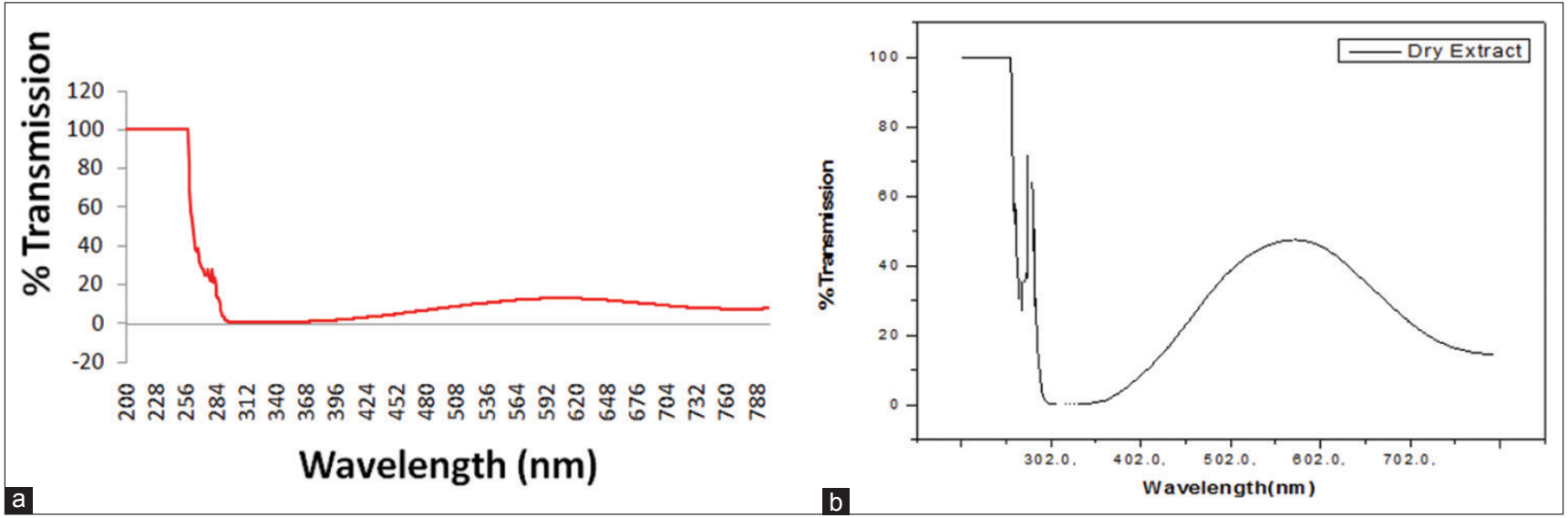

Fig. 2: Wavelength of fresh leaf (a) and dry lead (b) 


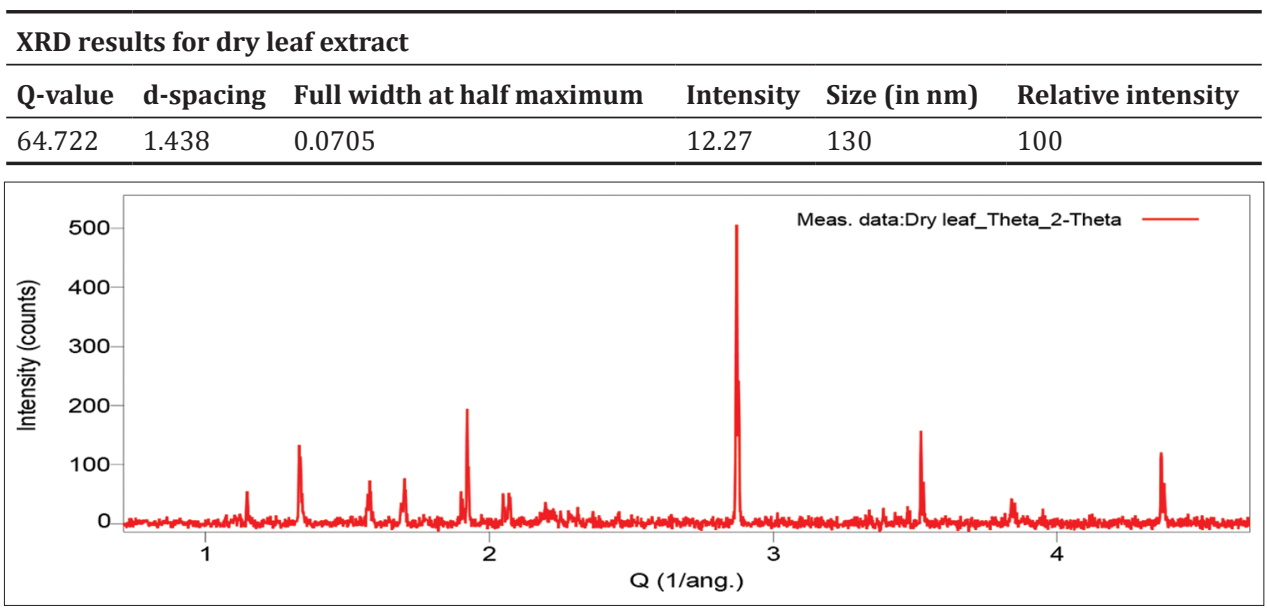

Fig. 3: X-ray diffraction of dry leaf extract

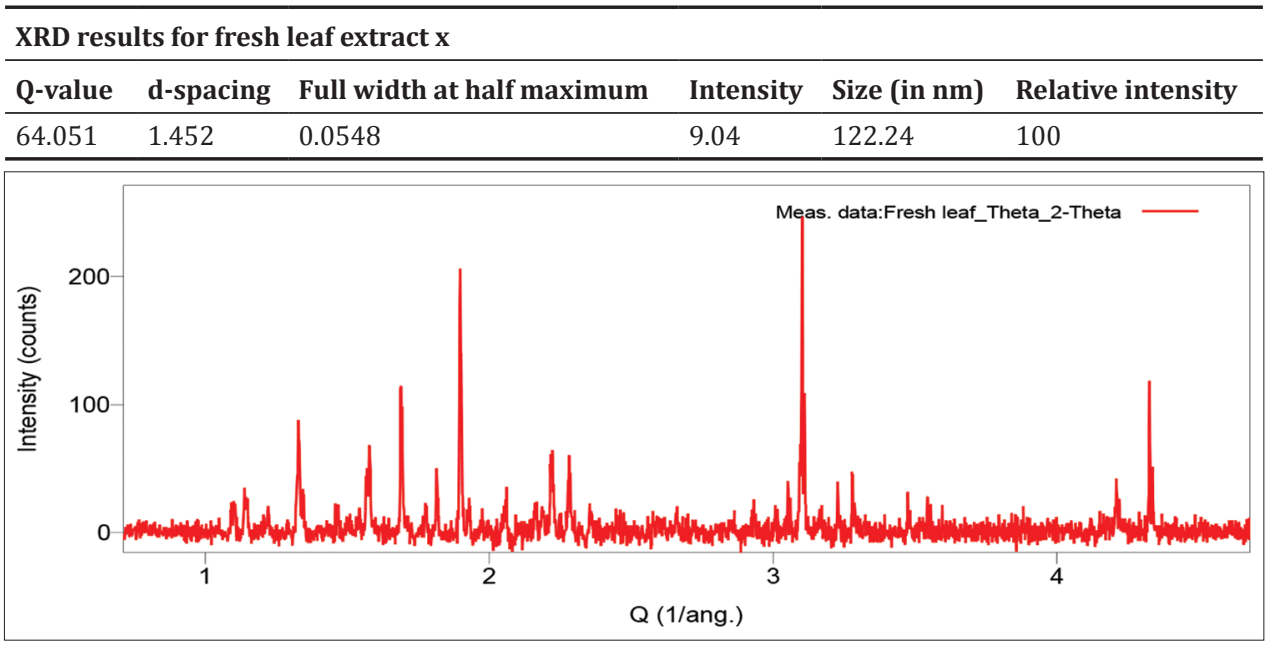

Fig. 4: X-ray diffraction of fresh leaf extract

crystallization of the bioorganic phase occurs on the surface of the CuNPs. Crystallite size of CuNPs as estimated from the full width at half maximum of the peak using Scherrer's formula exhibited average dry and fresh leaf extract particle size to be $130 \mathrm{~nm}$ and $122.24 \mathrm{~nm}$, respectively.

\section{SEM analysis}

SEM image provided the morphology of the CuNPs which was predominantly spherical and aggregated into the large irregular structures. SEM images of CuNP of dry leaf extract were $407.8 \mathrm{~nm}$ and $449.6 \mathrm{~nm}$ (Fig. 5) and of fresh leaf extract was $465.9 \mathrm{~nm}$ and $352.1 \mathrm{~nm}$ (Fig. 6). The size of the prepared nanoparticles was more than the size of nanoparticles which should be between 1 and $100 \mathrm{~nm}$.

\section{Antimicrobial analysis}

The antimicrobial activities against the pathogens were evaluated by the disc diffusion method through the zone of inhibition. After incubation, the zone of inhibition was measured to assess the inhibitory activity of the CuNPs. Synthesis of CuNPs was tested against the selected organisms such as $S$. aureus, B. subtilis, $P$. vulgaris, and P. aeruginosa, as shown in Figs. 7 and 8 . The result revealed that as the concentration of CuNps increased, the zone of inhibition also increased against the bacteria strain in dry leaves extract (Table 1) as well as in fresh leaves extract (Table 2). The antifungal activity of CuNPs was assayed against the selected fungi such as Penicillium spp., F. oxysporum, and A. niger. In the dry plant extract, all the fungi

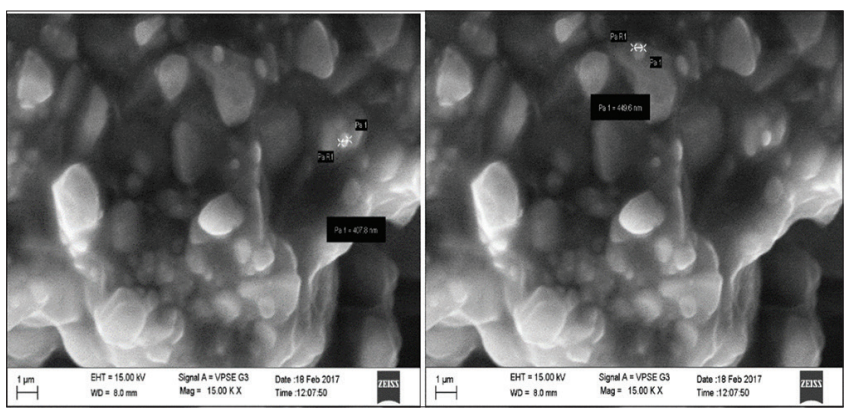

Fig. 5: Scanning electron microscopy images of dry leaf extract

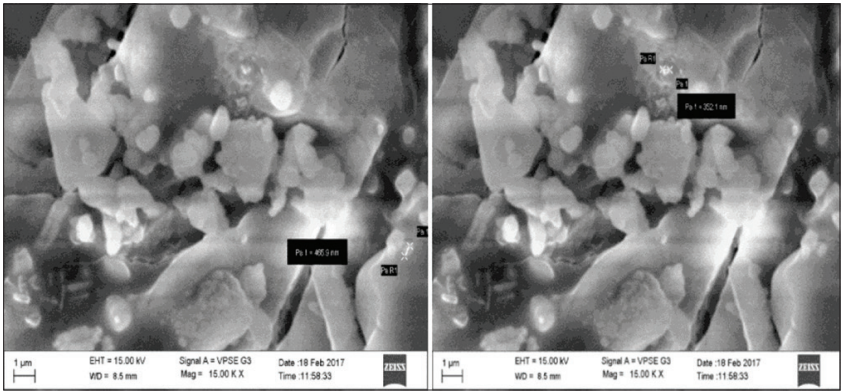

Fig. 6: Scanning electron microscopy images of fresh leaf extract 


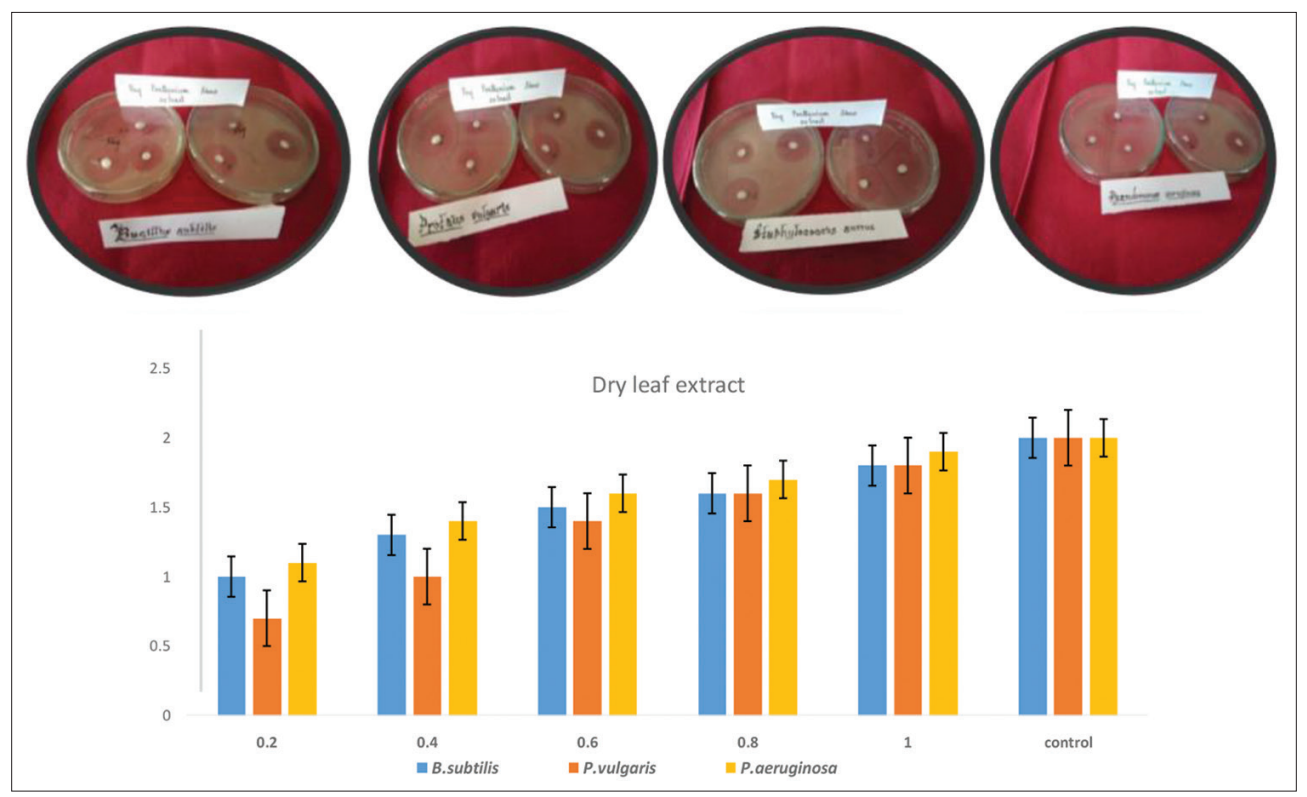

Fig. 7: Antibacterial activity of dry extract

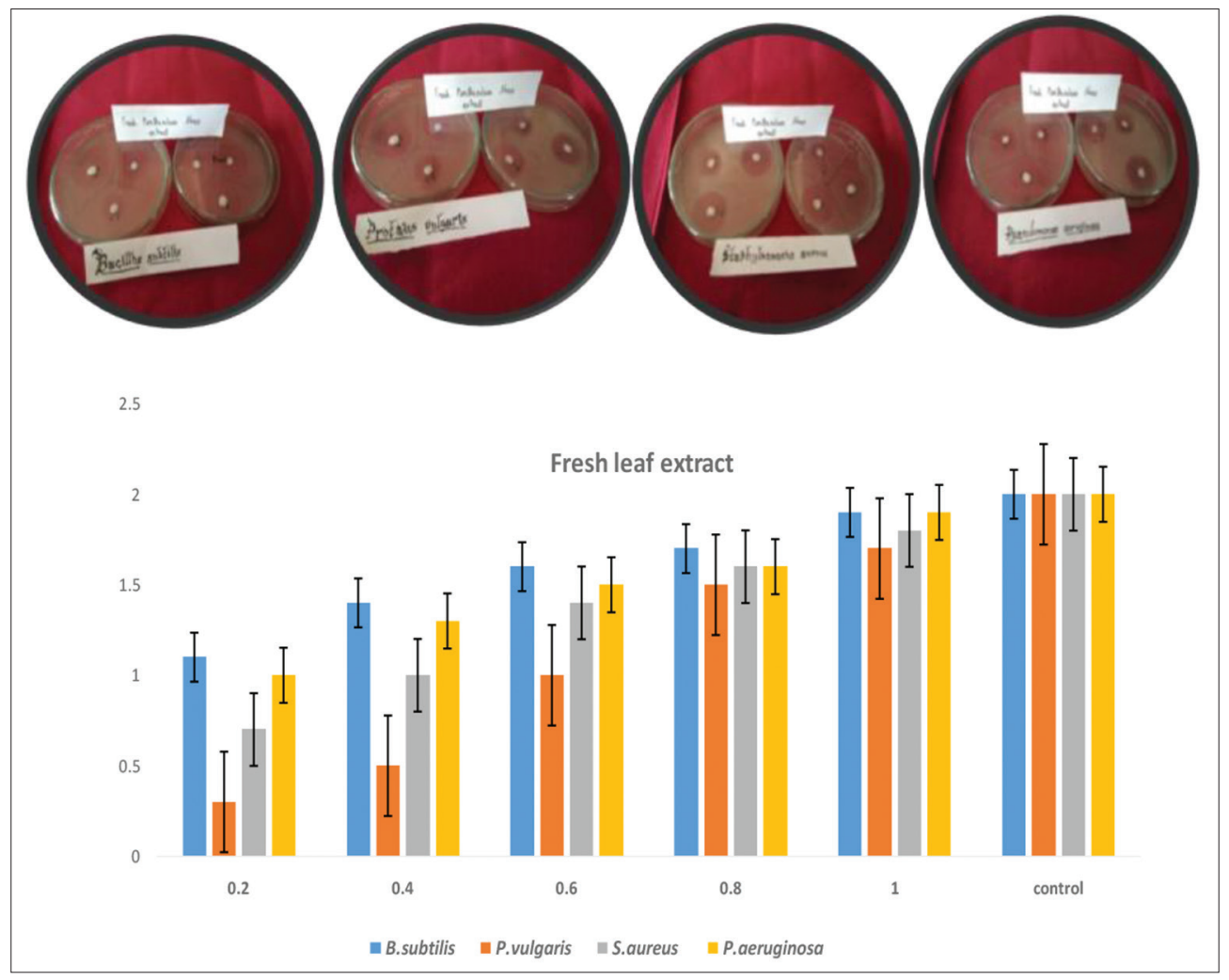

Fig. 8: Antibacterial activity of fresh extract

Table 1: Antibacterial activity of dry leaf extract

\begin{tabular}{llllll}
\hline S. No. & Concentration (mg) & Bacillus subtilis & Proteus vulgaris & Staphylococcus aureus & Pseudomonas aeruginosa \\
\hline 1. & 0.2 & 1.0 & 0.7 & 0.5 & 1.1 \\
2. & 0.4 & 1.3 & 1.0 & 1.2 & 1.4 \\
3. & 0.6 & 1.5 & 1.4 & 1.5 & 1.6 \\
4. & 0.8 & 1.6 & 1.6 & 1.6 & 1.7 \\
5. & 1.0 & 2.0 & 1.7 & 1.9 \\
6. & Positive control & 2.0 & 2.0 & 2.0 \\
\hline
\end{tabular}


exhibited zone of inhibition, whereas the A. niger showed very significant inhibition compare to all the other fungi (Table 3 and Fig. 9). In the fresh leaves extract, $F$. oxysporum showed a very good zone of inhibition. A. niger indicated the zone of inhibition based on the dose of concentration. Higher was the concentration better was the zone of inhibition (Table 4 and Fig. 10).

\section{DISCUSSION}

Using plant extracts as medicine are a traditional method, now with the biological reduction of metal, NPs using plant extract are a recent method. The use of plant extract is effective against various animals as well as plant pathogens; hence, it can also be used as fungicides or bactericides. The plant $P$. hysterophorus is highly invasive weed and a skin irritant. The phytotoxicity that causes the irritation is found to be minimized with the reduction of copper ions, supplied by copper sulfate solution to CuNPs. The ionic copper Cu (II) interacts with the thiol group of important phytoenzymes and activates them. Synthesis of $\mathrm{CuO}$ NPs was performed successfully using a chemical reduction method. The reaction occurs at room temperature and is generally completed within few minutes. In view of the number of different chemicals involved, the bioreduction process is relatively complex [13]. Drugs bound to nanoparticles have been claimed to have advantages compared with the conventional forms of the

Table 2: Antibacterial activity of fresh leaf extract

\begin{tabular}{llllll}
\hline S. No. & Concentration (mg) & Bacillus subtilis & Proteus vulgaris & Staphylococcus aureus & Pseudomonas aeruginosa \\
\hline 1. & 0.2 & 1.1 & 0.3 & 0.7 & 1.0 \\
2. & 0.4 & 1.4 & 0.5 & 1.0 & 1.3 \\
3. & 0.6 & 1.6 & 1.0 & 1.4 & 1.5 \\
4. & 0.8 & 1.7 & 1.5 & 1.8 & 1.6 \\
5. & 1.0 & 1.9 & 1.7 & 2.0 & 2.9 \\
6. & Positive control & 2.0 & 2.0 & & 2.0 \\
\hline
\end{tabular}

Table 3: Antifungal activity of dry leaf extract

\begin{tabular}{|c|c|c|c|c|}
\hline S. No. & Concentration (mg) & Aspergillus niger & Fusarium oxysporum & Penicillium spp. \\
\hline 1. & 10 & 1.0 & 0.4 & 0.8 \\
\hline 2. & 20 & 1.1 & 0.6 & 0.8 \\
\hline 3. & 30 & 1.2 & 0.7 & 1.4 \\
\hline 4. & 40 & 1.7 & 1.2 & 1.4 \\
\hline 5. & 50 & 2.0 & 2.0 & 1.6 \\
\hline 6. & Positive control & 1.0 & 1.0 & 1.0 \\
\hline
\end{tabular}

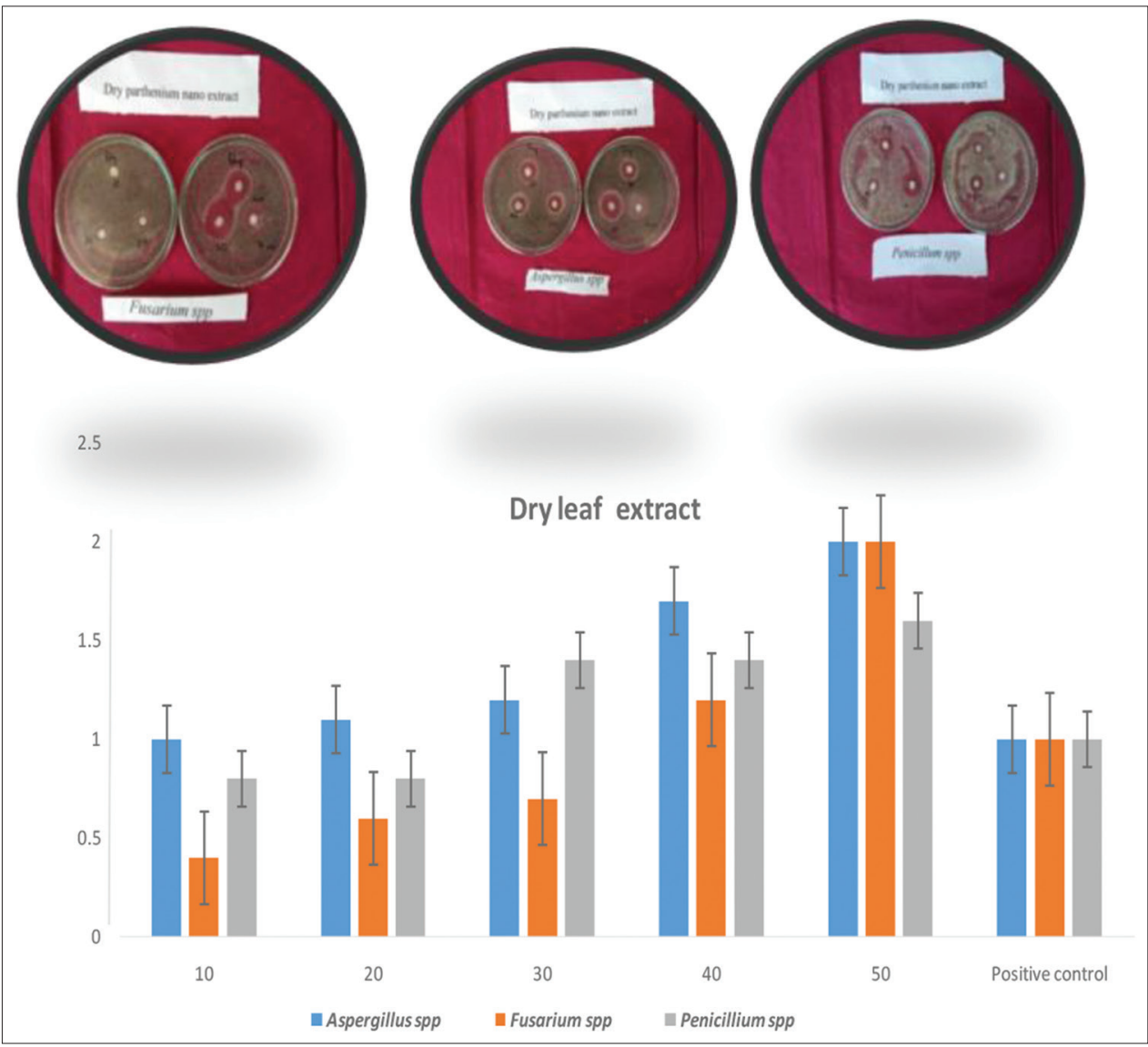

Fig. 9: Antifungal activity of dry extract 
Table 4: Antifungal activity of fresh leaf extract

\begin{tabular}{|c|c|c|c|c|}
\hline S. No. & Concentration (mg) & Aspergillus niger & Fusarium oxysporum & Penicillium spp. \\
\hline 1. & 10 & 0.0 & 0.6 & 0.0 \\
\hline 2. & 20 & 0.0 & 0.8 & 0.4 \\
\hline 3. & 30 & 0.1 & 0.9 & 1.0 \\
\hline 4. & 40 & 1.6 & 1.0 & 0.6 \\
\hline 5. & 50 & 1.7 & 1.7 & 0.7 \\
\hline 6. & Positive control & 1.0 & 1.0 & 1.0 \\
\hline
\end{tabular}

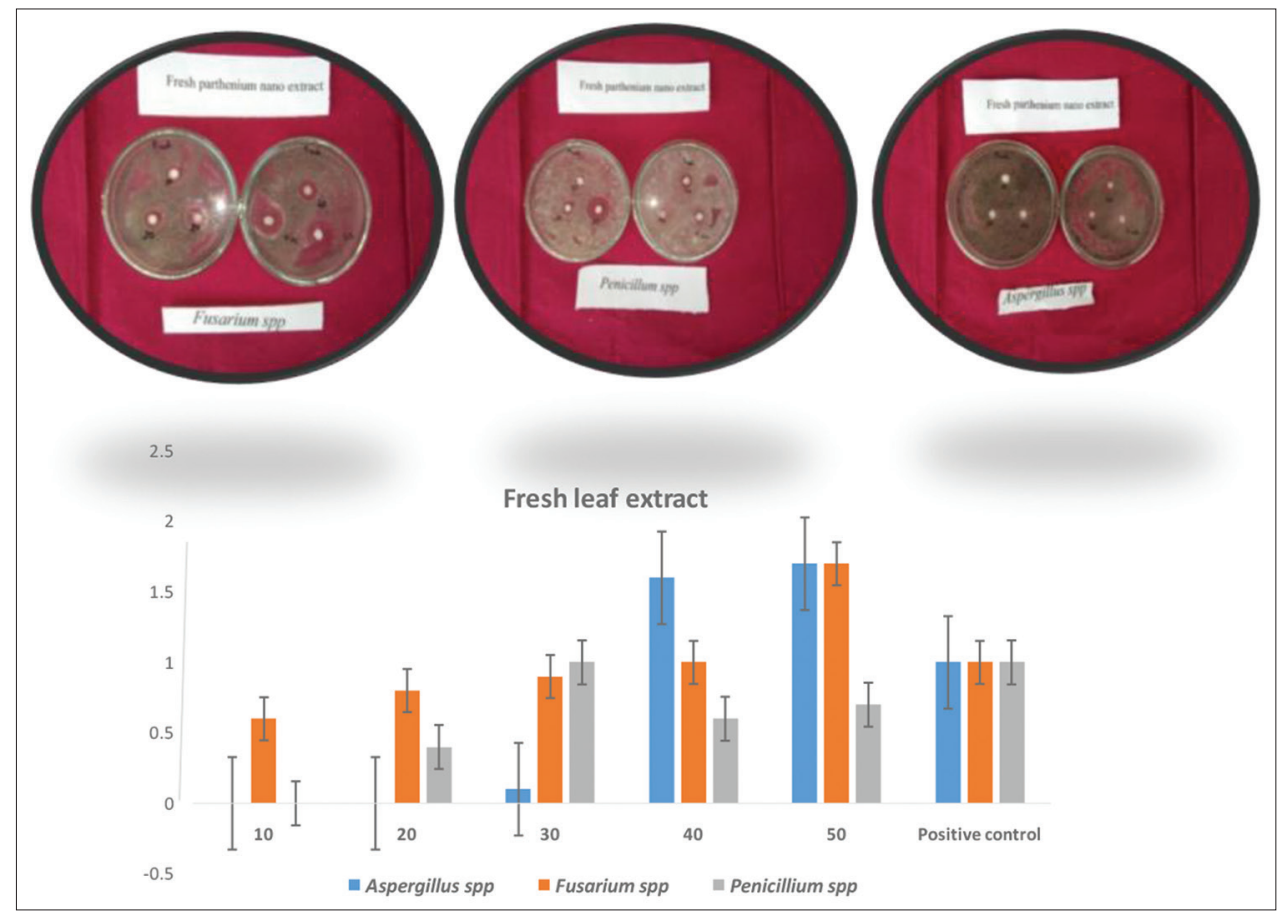

Fig. 10: Antifungal activity of fresh extract

drugs [14]. The CuO NPs showed remarkable antibacterial activity against Streptococcus sp. and Staphylococcus sps. activity against Streptococcus sps. and Staphylococcus sps. The studies reveal the mechanism of the bactericidal action of NPs [15]. The biologically reduced CuNPs using dry and fresh Parthenium leaf extract were found to be toxic to selected pathogenic bacteria and fungi. By this method, Parthenium can be used for novel causes such as drug designing and plant bactericides and fungicides. The CuNPs of dry and fresh leaf extracts showed higher toxicity because the leaf extracts synthesized a higher concentration of CuNPs as it is the site of photosynthesis and the availability of more $\mathrm{H}+$ ions to reduce copper sulfate to CuNPs. The molecular basis for the bioreduction of metal ions into MNPs and in this case copper ions into CuNPs is speculated that the organic matrix contains metal-binding proteins that provide amino acid moieties that serve as the nucleation sites. This present research also reveals a simple, rapid, and economical way of synthesizing CuNPs and their capability of rendering antimicrobial efficiency and provides a medicinal purpose to this particular plant.

The antibacterial potentials of the CuNPs study have indicated significant inhibition activity against $S$. aureus, B. subtilis, P. vulgaris, and $P$. aeruginosa at different concentrations in compare to fungi species. The zone of inhibition was based on the increase in the concentration of the plant extracts of $P$. hysterophorus. As per the results obtained, the highest concentration shows maximum inhibition. Hence, it can be used in the treatment of infectious diseases caused by tested strains and potential antimicrobial agents may be developed. However, further studies must be performed to identify the specific principles responsible for the antimicrobial activity of $P$. hysterophorus.

\section{CONCLUSION}

This greener approach toward the synthesis of CuNPs, using plant leaf material as reducing and capping agent, has many advantages such as ease with which the process can be scaled up, economic viability, environmentally benign, and renewable, there is no need to use high pressure, energy, temperature, and toxic chemicals. Applications of eco-friendly CuNPs in bactericidal, wound healing, and other medical and electronic applications are potentially exciting for their large-scale synthesis. Toxicity of CuNPs on human pathogen bacteria opens a door for a new range of antibacterial agents.

\section{ACKNOWLEDGMENT}

The authors are grateful to the authorities of Yuvaraja's College and Teresian College, Mysore, for providing the required facilities.

\section{AUTHORS' CONTRIBUTIONS}

Pruthvi and Rohini have prepared the manuscript under the guidance of Dr. Mahesh.

\section{CONFLICTS OF INTEREST}

The authors declare that they have no conflicts of interest.

\section{REFERENCES}

1. Shankar SS, Rai A, Ahmad A, Sastry M. Rapid synthesis of Au, Ag, and bimetallic $\mathrm{Au}$ core- $\mathrm{Ag}$ shell nanoparticles using neem (Azadirachta indica) leaf broth. J Colloid Interface Sci 2004;275:496-502.

2. Kumar V, Yadav SK. Plant-mediated synthesis of silver and gold 
nanoparticles and their applications. J Chem Technol Biotechnol 2009;84:151-7.

3. Mukunthan K, Balaji S. Cashew apple juice (Anacardium occidentale L.) speeds up the synthesis of silver nanoparticles. Int J Green Nanotechnol 2012;4:71-9.

4. Sahoo SK, Parveen S, Panda JJ. The present and future of nanotechnology in human health care. Nanomedicine 2007;3:20-31.

5. Mohanraj Y, Chen A. Nanoparticles a review. Trop J Pharm Res 2007;5:561-73.

6. Panyam J, Labhasetwar V. Biodegradable nanoparticles for drug and gene delivery to cells and tissue. Adv Drug Deliv Rev 2003;55:329-47.

7. Han S, Liu Y, Chang A. Cytoplasmic Hsp70 promotes ubiquitination for endoplasmic reticulum-associated degradation of a misfolded mutant of the yeast plasma membrane ATPase, PMA1. J Biol Chem 2007;282:26140-9.

8. Perelshtein I, Applerot G, Perkas N, Wehrschuetz-Sigl E, Hasmann A, Guebitz $\mathrm{G}$, et al. CuO-cotton nanocomposite: Formation, morphology, and antibacterial activity. Surf Coat Technol 2009;204:54-7.

9. Valodkar M. Synthesis and anti-bacterial activity of $\mathrm{Cu}, \mathrm{Ag}$ and $\mathrm{Cu}-\mathrm{Ag}$ alloy nanoparticles: A green approach. Mater Res Bull 2011;46:384-9.

10. Kattumuri V, Katti K, Bhaskaran S, Boote EJ, Casteel SW, Fent GM, et al. Gum Arabic as a phytochemical construct for the stabilization of gold nanoparticles: In vivo pharmacokinetics and X-ray-contrastimaging studies. Small 2007;3:333-41.

11. Virkutyte J, Varma RS. Green synthesis of metal nanoparticles: Biodegradable polymers and enzymes in stabilization and surface functionalization. J Chem Sci 2011;2:837.

12. Wagner MC, Molnar EE, Molitoris BA, Goebl MG. Loss of the homotypic fusion and vacuole protein sorting or golgi-associated retrograde protein vesicle tethering complexes results in gentamicin sensitivity in the yeast Saccharomyces cerevisiae. Antimicrob Agents Chemother 2006;50:587-95.

13. Huang K, Ferrin-O'Connell I, Zhang W, Leonard GA, O'Shea EK, Quiocho FA. Structure of the Pho85-Pho80 CDK-cyclin complex of the phosphate-responsive signal transduction pathway. Mol Cell 2007;28:614-23.

14. Mary S, Mahesh MK. Determination of antibacterial and antifungal activities of endemic tree fern Cyathea nilgirensis plant extracts. Int $\mathrm{J}$ Curr Res 2015;7:18276-9.

15. Yoon KY, Hoon Byeon J, Park JH, Hwang J. Susceptibility constants of Escherichia coli and Bacillus subtilis to silver and copper nanoparticles. Sci Total Environ 2007;373:572-5. 